\title{
Age-related morphologic changes alter robustness of neuronal function
}

\author{
Aniruddha Yadav ${ }^{1,2^{*}}$, Christina M Weaver ${ }^{3}$, Yuan Z Gao ${ }^{1,2}$, Jennifer I Luebke ${ }^{4}$, Patrick R Hof ${ }^{1,2}$ \\ From Nineteenth Annual Computational Neuroscience Meeting: CNS*2010 \\ San Antonio, TX, USA. 24-30 July 2010
}

Aging and neurodegenerative disorders significantly alter both neuronal function and morphology. We have shown recently that, if uncompensated by altered ion channel distributions, morphologic changes in aging increase neuronal excitability [1]. Different combinations of active conductances can evoke similar firing patterns, giving rise to functional homeostasis. To elucidate possible mechanisms of homeostasis in the parameter space defined by ionic conductances, we recently utilized thermal random walks to identify regions (an ensemble) within which the functional output of a neuron is maintained. Typically a number of such regions, which are topologically disconnected from each other, are identified. The eigenvalues and eigenvectors of the ensemble covariance matrix serve as measures for its size and orientation, and the capacity for functional homeostasis or robustness is measured by the set of largest (robust) eigenvalues and corresponding eigenvectors. In this study, we explore how functional homeostasis might be affected by the kinds of morphologic changes that occur with aging or neurodegenerative disorders. To this end, we extract the change in orientation and extent of the ensembles found for the original model by subjecting it to three kinds of perturbations involving morphology and desired firing properties. First, we mimic age-related morphologic degeneration by reducing the number of spines in the original neuron. Second, we increase the number of spines in the original neuron, mimicking a younger one. The third perturbation also involves increased spines, however, the target firing rate is reduced so as to also mimic the electrophysiology of a younger neuron. We find that, for all the perturbations considered, the initial ensembles remain topologically disconnected, although they change in size and orientation. In particular, while the sensitive eigendirections

${ }^{1}$ Department of Neuroscience, Mount Sinai School of Medicine, New York, NY, USA remain unchanged, the robust eigendirections change in orientation, suggesting that functional robustness of a neuron is determined largely by its morphology. Consequently, possible homeostatic mechanisms, that enable a young neuron to maintain function, are able to do so less efficiently as the neuron undergoes age related morphologic changes .This is so because a homeostatic mechanism would be more effective if it were to orient itself along the robust eigendirections of the ensembles, and become less so as the robust directions change due to morphologic changes. Although morphologic perturbations drastically change robust eigendirections, preliminary evidence indicates that these directions may be restored through appropriate compensatory changes in functional output. In conclusion, the need to maintain functional robustness may be responsible for altered function as a neuron ages.

\section{Acknowledgements}

Supported by NIH grants DC05669, NS13742 and MH071818.

\section{Author details}

'Department of Neuroscience, Mount Sinai School of Medicine, New York, NY, USA. ${ }^{2}$ Computational Neurobiology and Imaging Center, Mount Sinai School of Medicine, New York, NY, USA. ${ }^{3}$ Department of Mathematics and Computer Science, Franklin \& Marshall College, Lancaster, PA, USA.

${ }^{4}$ Department of Anatomy and Neurobiology, Boston University, Boston, MA, USA.

\section{Published: 20 July 2010}

\section{Reference}

1. Kabaso D, Coskren PJ, Henry BI, Hof PR, Wearne SL: The electrotonic structure of pyramidal neurons contributing to prefrontal cortical circuits in macaque monkeys is significantly altered in aging. Cereb Cortex 2009, 19:2248-2268.

doi:10.1186/1471-2202-11-S1-P140

Cite this article as: Yadav et al: Age-related morphologic changes alter robustness of neuronal function. BMC Neuroscience 2010 11(Suppl 1):P140. 